\title{
Monitoring early changes in rectal tumor morphology and volume during 5 weeks of preoperative chemoradiotherapy
}

Citation for published version (APA):

Lambregts, D. M. J., Yassien, A. B., Lahaye, M. J., Betgen, A., Maas, M., Beets, G. L., van der Heide, U. A., van Triest, B., \& Beets-Tan, R. G. H. (2018). Monitoring early changes in rectal tumor morphology and volume during 5 weeks of preoperative chemoradiotherapy: An evaluation with sequential MRIs. Radiotherapy and Oncology, 126(3), 431-436. https://doi.org/10.1016/j.radonc.2017.12.024

Document status and date:

Published: 01/03/2018

DOI:

10.1016/j.radonc.2017.12.024

Document Version:

Publisher's PDF, also known as Version of record

\section{Document license:}

Taverne

Please check the document version of this publication:

- A submitted manuscript is the version of the article upon submission and before peer-review. There can be important differences between the submitted version and the official published version of record.

People interested in the research are advised to contact the author for the final version of the publication, or visit the DOI to the publisher's website.

- The final author version and the galley proof are versions of the publication after peer review.

- The final published version features the final layout of the paper including the volume, issue and page numbers.

Link to publication

\footnotetext{
General rights rights.

- You may freely distribute the URL identifying the publication in the public portal. please follow below link for the End User Agreement:

www.umlib.nl/taverne-license

Take down policy

If you believe that this document breaches copyright please contact us at:

repository@maastrichtuniversity.nl

providing details and we will investigate your claim.
}

Copyright and moral rights for the publications made accessible in the public portal are retained by the authors and/or other copyright owners and it is a condition of accessing publications that users recognise and abide by the legal requirements associated with these

- Users may download and print one copy of any publication from the public portal for the purpose of private study or research.

- You may not further distribute the material or use it for any profit-making activity or commercial gain

If the publication is distributed under the terms of Article $25 \mathrm{fa}$ of the Dutch Copyright Act, indicated by the "Taverne" license above, 
MRI in rectal cancer

\title{
Monitoring early changes in rectal tumor morphology and volume during 5 weeks of preoperative chemoradiotherapy - An evaluation with sequential MRIs
}

\author{
Doenja M.J. Lambregts ${ }^{\mathrm{a}, *}$, Ahmed Bani Yassien ${ }^{\mathrm{b}}$, Max J. Lahaye ${ }^{\mathrm{a}}$, Anja Betgen ${ }^{\mathrm{b}}$, Monique Maas ${ }^{\mathrm{a}}$, \\ Geerard L. Beets ${ }^{c, d}$, Uulke A. van der Heide ${ }^{b}$, Baukelien van Triest ${ }^{b}$, Regina G.H. Beets-Tan ${ }^{\text {a,d }}$

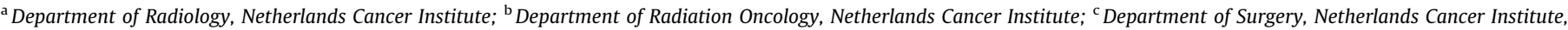 \\ Amsterdam, The Netherlands; ${ }^{\mathrm{d}}$ GROW School for Oncology and Developmental Biology - University of Maastricht, Maastricht, The Netherlands
}

\section{A R T I C L E I N F O}

\section{Article history:}

Received 15 November 2017

Received in revised form 18 December 2017

Accepted 27 December 2017

Available online 16 January 2018

\section{Keywords:}

Rectal cancer

Early response

Radiotherapy

MRI

Dose escalation

\begin{abstract}
A B S T R A C T
Purpose: To assess early changes in rectal tumor volume and morphology on sequential MRIs performed during 5 weeks of chemoradiotherapy.

Materials and methods: Thirteen patients underwent weekly T2W-MRI during 5 weeks of preoperative radiotherapy (total $50 \mathrm{~Gy}$ ), starting after the first week of radiation. Two radiologists visually evaluated tumor volume and morphology and one reader manually segmented tumors for each time point to quantitatively calculate tumor volumes. Evolution in tumor volume/morphology was assessed over time and compared between good responders (tumor regression grade (TRG) 1-2) and poor responders (TRG 3-5). Results: Tumor volumes decreased significantly during radiation. Early signs of response were also visually apparent: in the majority of good responders an early fibrotic transformation (week 2-3) as well as a visually estimated early volume reduction of $>1 / 3$ (week 1-2), was observed while these early changes only occurred in a minority of poor responders.

Conclusion: Results of this exploratory pilot study suggest that changes in rectal tumor morphology (fibrosis) and volume can already be observed early during radiation, both when measured quantitatively and when assessed visually. These changes appear to be indicative of the final treatment outcome.
\end{abstract}

(C) 2018 Elsevier B.V. All rights reserved. Radiotherapy and Oncology 126 (2018) 431-436

\section{Introduction}

According to current guidelines, standard treatment for locally advanced rectal cancer (LARC) consists of a long course of neoadjuvant chemoradiotherapy (CRT) followed by surgical resection. In recent years there has been increasing attention for the concept of 'organ preservation', in particular 'watchful waiting'. In a watchful waiting policy, patients who have a clinical complete tumor regression after CRT are deferred from surgery and followed intensely. In case of a tumor regrowth, patients may still undergo salvage TME, whereas in patients that show a sustained complete response, surgery can be definitively evaded thereby avoiding complications and morbidity related to resection [1-4]. A pooled analysis of $>3000$ patients showed that approximately $15 \%$ of LARC patients achieve a complete response after routine CRT with a radiation dose of 45-50.4 Gy [5], indicating that only a minority of

\footnotetext{
* Corresponding author at: Department of Radiology, Netherlands Cancer Institute, P.O. Box 90203, 1006 BE Amsterdam, The Netherlands.

E-mail address: d.lambregts@nki.nl (D.M.J. Lambregts).
}

patients will be potential candidates for watchful waiting. In order to increase these numbers and ultimately offer more patients the benefits of organ preservation, efforts are being undertaken to maximize response rates by optimizing neoadjuvant treatment schemes, for example by means of radiation dose escalation to the tumor ('boosting'). A meta-analysis showed that an escalated preoperative dose of $\geq 60 \mathrm{~Gy}$ may lead to increased pCR rates of over $20 \%$ [6].

Two important challenges to allow meaningful adaptive neoadjuvant treatment are to identify the right patients and determine the proper timing to alter or intensify treatment in order to maximize therapeutic effects. Patients who show a response to routine CRT are more likely to benefit from further dose escalation to increase the chance of a complete response. Conversely, for patients who do not respond or respond very poorly to routine CRT, an extra dose will unlikely lead to therapeutic benefit while patients are still subjected to added toxicity. To discriminate between these patient groups, an early assessment of how well patients will respond to treatment would be of great value. Moreover, knowledge of the timeline of early tumor regression 
can be used to determine the best time point to perform an early response evaluation.

Although numerous studies have focused on the use of imaging to evaluate response after completion of long course CRT, limited data are available on imaging for early response assessment. Several reports have evaluated the use of Positron Emission Tomography (PET) to measure response 1-2 weeks after onset of treatment, showing that early changes in tumor glucose metabolism may be useful for early response assessment [7,8]. PET is, however, not routinely used in clinics for the local staging and response assessment of rectal tumors. MRI is to date the most widely used imaging modality. There is some evidence that functional MRI (e.g. diffusion-weighted and dynamic contrastenhanced MRI) performed early during treatment can aid in predicting the final treatment response [9-12], but so far only a few studies have investigated the use of routine morphological MRI and mainly at one or two time points during treatment $[10,13,14]$. One previous report by van de Begin et al. calculated gross tumor volumes on multiple sequential MRIs performed before, during and after preoperative chemoradiotherapy and concluded that rectal tumor regression mainly occurs during the first half of the preoperative CRT course [15].

Aim of this study was to add to these limited previous data and explore (in a pilot analysis) how rectal tumor morphology and volume change early during radiation treatment with sequential 'routine' T2-weighted MRIs performed during 5 weeks of radiotherapy in order to obtain a better understanding of early morphological signs of rectal tumor regression

\section{Materials and methods}

This study was approved by the local institutional review board. All patients provided written informed consent.

\section{Patients}

From October 2013 till May 2015, thirteen patients with locally advanced rectal cancer (cT3-4 and/or cN+), scheduled to undergo a long course of preoperative chemoradiotherapy were prospectively enrolled to undergo weekly MRI during their 5 weeks of radiation treatment. Inclusion criteria consisted of biopsy proven rectal adenocarcinoma and routine long course neoadjuvant treatment which according to our institutional guideline consisted of $50 \mathrm{~Gy}$ in 25 fractions on weekdays for 5 weeks combined with Capecitabine $825 \mathrm{mg} / \mathrm{m} 2$ BID on radiation treatment days.

\section{MR imaging}

As part of the study protocol patients underwent weekly MRIs ( 5 in total) during the 5 weeks of radiation treatment using a standardized protocol. The first MRI was performed on the first weekday after the first week of radiation, the following MRIs were routinely done on the first weekday of radiation (before the start of treatment), with the final MRI performed after completion of the fifth week of radiation. Images were acquired on a 3.0 T MRI system (Achieva, Philips Medical Systems, Best, The Netherlands) using a phased array surface coil. The MR sequences used for the current study consisted of 2D T2-weighted TSE sequences in 3 orthogonal directions (true sagittal, transverse and coronal plane) with the following scan parameters (for the transverse sequence): $\mathrm{TR} \pm 3200-6200$ (shortest), TE $120 \mathrm{msec}, 90^{\circ}$ flip angle, 30 echo train length, $1 \mathrm{NSA}, 0.80 \times 0.84 \times 3.0 \mathrm{~mm}$ acquired voxel size, 27-49 slices, 2:26-4:22 minutes acquisition time. Outside the scope of the study, patients also underwent a primary staging MRI before treatment and a restaging MRI after completion of CRT (and a waiting interval) as part of routine clinical diagnostic procedures. These clinical MRI examinations were partly performed elsewhere (in referring hospitals) and not according to the standardized study protocol.

\section{Visual evaluation}

Two expert radiologists (DL and $\mathrm{ML}$; each with $>8$ years of specific experience in reading rectal MRI) in consensus visually assessed the size/volume and morphology of the rectal tumor for each patient on the primary staging MRI performed before treatment and studied their evolution over time for the 5 study scans performed during radiation treatment. The two readers evaluated:

- the morphology of the primary tumor before onset of treatment (solid vs. mucinous, polypoid vs. (semi)circular, regular vs. irregular tumor boundaries)

- when, i.e. in which week of treatment, a fibrotic transformation became visually apparent (see Fig. 1). Fibrotic transformation was defined as a reduction in signal on T2-weighted MRI occurring within the rectal wall / tumor bed.

- when, i.e. in which week of treatment, a substantial volume reduction (i.e., a visually estimated decrease of $\pm 1 / 3$ or more in tumor volume) became apparent (see Fig. 1).

When there was any disagreement between the two readers, a third expert with $>15$ years of rectal MRI experience (RB-T) was consulted to reach a final decision.

\section{Quantification of tumor volumes}

One of the readers (DL) manually delineated the tumor boundaries on each consecutive slice containing tumor (see Fig. 2) in order to calculate whole tumor volumes. Delineations were performed using the free open source platform 3D Slicer (www. slicer.org) [16].

\section{Data analyses}

Statistical analyses were performed using IBM SPSS version 22 (IBM ${ }^{\circledR}$ Corps., Darmonk, NY, USA). The main outcome was the final tumor response as assessed at histopathology after surgery using the tumor regression grade (TRG) of Mandard [17]. Patients with a TRG 1-2 were classified as good responders; patients with a TRG 3-5 were considered poor responders. Initial tumor morphology and the timeline of response (as visually assessed) were compared between good and poor responders using descriptive statistics. Absolute tumor volumes ( \pm standard deviations) as well as the percentage volume decrease after each week were plotted and compared between the five different time points as well as between patients from the poor vs. good response group using non-parametric paired and independent tests respectively (because data were not normally distributed). $P$-values $<0.05$ were considered statistically significant.

\section{Results}

\section{Patient characteristics}

Nine patients were male, 4 were female. Median age at the time of diagnosis was 65 years (range 49-75). TRG scores were available in $10 / 13$ patient (TRG1-2 in $n=3$; TRG $3-5$ in $n=7$ ). For three patients, the TRG was not available. Two of these three patients were classified as poor responders ( 1 underwent palliative care after CRT due to progressive metastasized disease with obvious signs of vital residual tumor on the final post-CRT MRI, 1 had a yT2N1 residual tumor at histopathology with a clear residual tumor mass on the final post-CRT MRI performed just before 

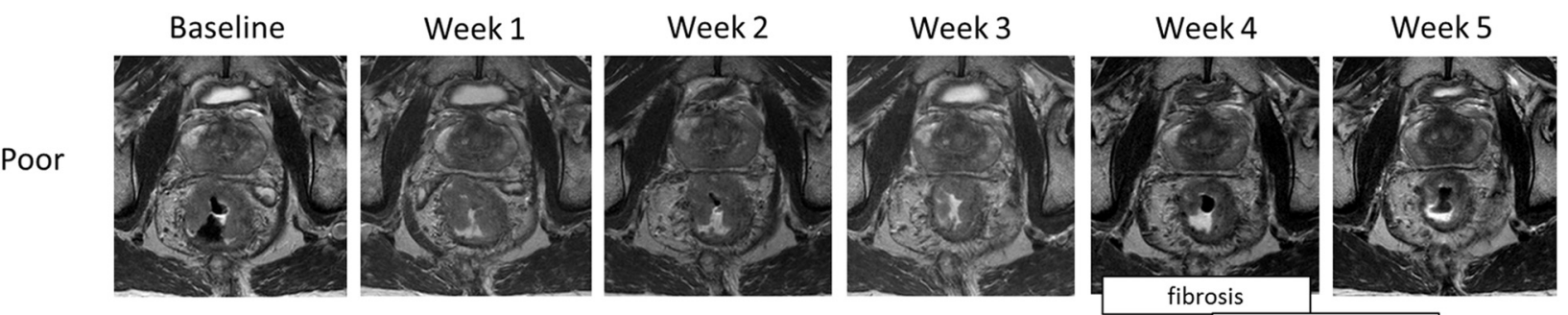

volume reduction
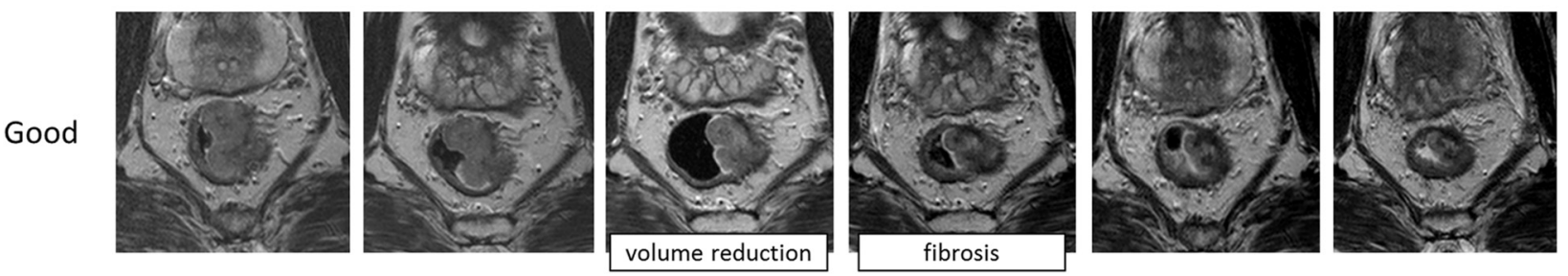

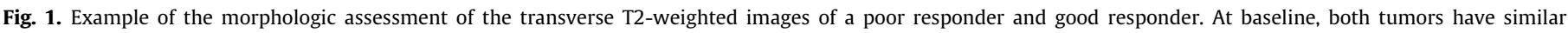

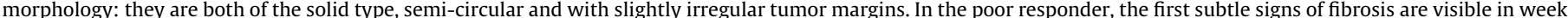

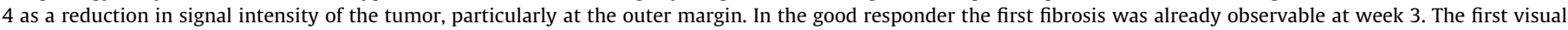
signs of a substantial ( $\pm 1 / 3$ or more) volume reduction became apparent in week $4-5$ for the poor responder and in week 2 for the good responder.

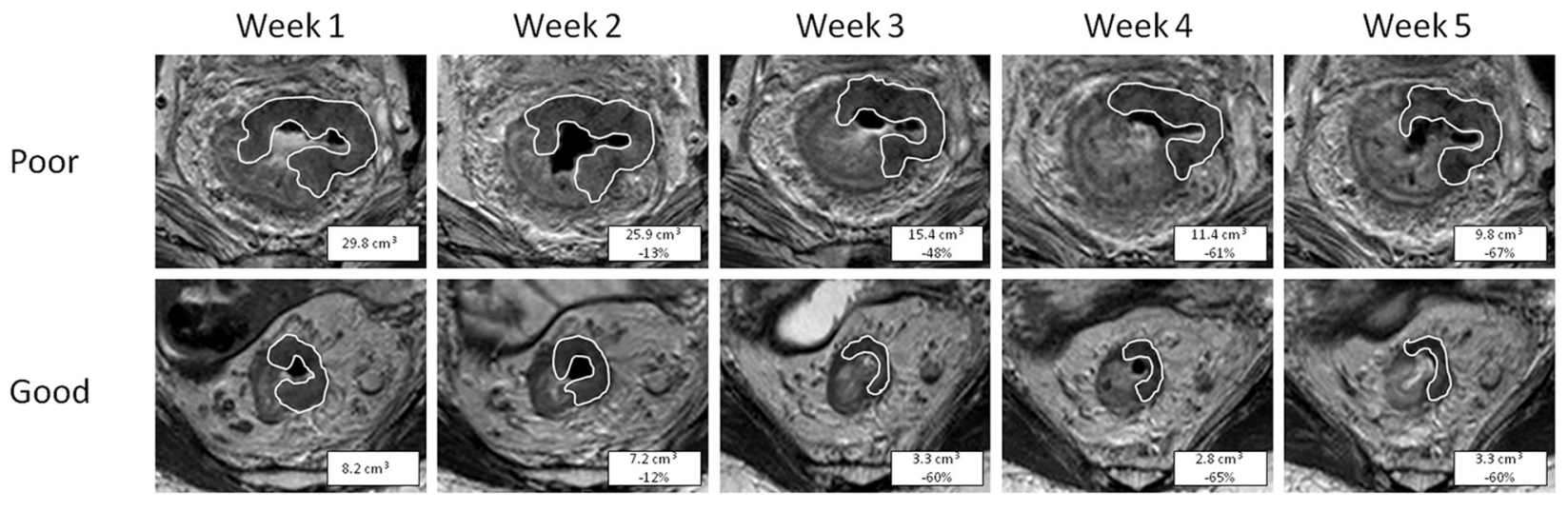

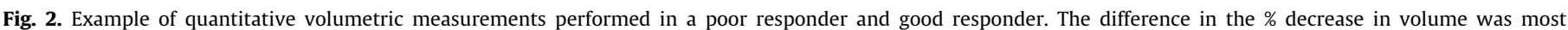

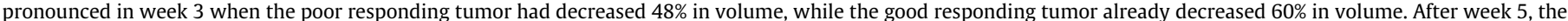

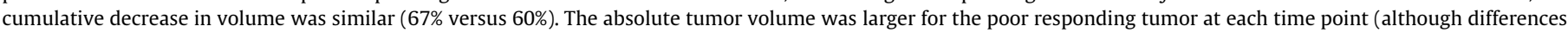
did not reach statistical significance).

surgery). The third patient was classified as a good responder (this patient was a clinical complete responder, managed according to a watchful waiting strategy since May 2015 with strong clinical evidence of a sustained complete tumor regression at multiple follow-up MRI and endoscopy examinations and a recurrence free follow-up interval of $>2$ years to date). This added up to a total of 4 good responders and 9 poor responders.

\section{Tumor morphology and visual assessment of response}

Imaging examples of a patient from the poor and good response group, respectively, are provided in Fig. 1. Findings for the primary tumor morphology and visual (morphologic) assessment of response are summarized in Table 1 for each patient.

The majority (12/13) of tumors was of the solid type, 1 patient (in the poor response group) presented with a largely solid but partly mucinous tumor. The distribution of primarily polypoid, circular and semi-circular tumors was similar among patients from the poor and good response group. In the good response group, $1 / 4$ tumors (25\%) primarily showed irregular margins, in the poor response group this was the case for $3 / 9$ tumors (33\%).
A fibrotic transformation was visible relatively early (after week $2-3)$ in $3 / 4(75 \%)$ of the good responders versus $4 / 9$ (44\%) of the poor responders. In $3 / 4(75 \%)$ of the good responders, a substantial volume reduction (estimated $1 / 3$ or more) became visually apparent early after week 1-2; in the poor response group this occurred in only $1 / 9(11 \%)$ of patients. In the remaining poor responders a substantial volume reduction was visually observed late after the 4 th to 5 th week of radiation (5/9: $56 \%$ ) or not at all (3/9: $33 \%)$.

The two readers reached full consensus in all 13 cases (and the independent third expert reader agreed with the findings of the two initial readers in all cases).

\section{Quantitative volume regression during radiotherapy}

Imaging examples of the quantitative volume evolution of two patients from the poor and good response group, respectively, are provided in Fig. 2. Absolute tumor volumes per week are provided in Table 1 for each patient. The graphs in Fig. 3 show the absolute mean ( $\pm \mathrm{SD}$ ) tumor volumes (Fig. $3 a$ ), and the \% volume decrease per week (Fig. $3 b$ ) with results presented separately for the good and poor responders. 
Table 1

Volumetric measurements and morphologic tumor assessment for each study patient.

\begin{tabular}{|c|c|c|c|c|c|c|c|c|c|c|c|}
\hline \multirow[b]{2}{*}{$\mathrm{Pt}$} & \multirow[b]{2}{*}{ Response } & \multicolumn{5}{|c|}{ Tumor Volume $\left(\mathrm{cm}^{3}\right)$} & \multicolumn{3}{|c|}{ Morphology before treatment } & \multicolumn{2}{|c|}{ Visual response assessment } \\
\hline & & $\begin{array}{c}\text { Wk } \\
1\end{array}$ & $\begin{array}{l}\text { Wk } \\
2\end{array}$ & $\begin{array}{l}\text { Wk } \\
3\end{array}$ & $\begin{array}{c}\text { Wk } \\
4\end{array}$ & $\begin{array}{l}\text { Wk } \\
5\end{array}$ & $\begin{array}{l}\text { Solid vs. } \\
\text { Mucinous }\end{array}$ & $\begin{array}{l}\text { Polypoid vs. } \\
\text { (semi-)circular }\end{array}$ & $\begin{array}{l}\text { Regular vs. } \\
\text { Irregular }\end{array}$ & $\begin{array}{l}\text { First subjective sign of } \\
\text { substantial volume } \downarrow\end{array}$ & $\begin{array}{l}\text { First subjective sign } \\
\text { of fibrosis }\end{array}$ \\
\hline 1 & Good & 8.2 & 7.2 & 3.3 & 2.8 & 3.3 & Solid & Semi-circular & Regular & Wk $1-2$ & Wk 2-3 \\
\hline 2 & Good & 75.3 & 45.4 & 30.7 & 25.0 & - & Solid & Semi-circular & Regular & Wk 2 & Wk 2-3 \\
\hline 3 & Good & - & 10.0 & 7.0 & 5.4 & 4.4 & Solid & Circular & Irregular & None & Wk 3-4 \\
\hline 4 & Good & 16.0 & 10.8 & 7.0 & 5.4 & 4.4 & Solid & Polypoid & Regular & Wk 2 & Wk 3 \\
\hline 5 & Poor & 30.4 & 30.2 & 25.8 & 21.4 & 18.1 & $\begin{array}{l}\text { Partly } \\
\text { mucinous }\end{array}$ & Semi-circular & Irregular & None & Wk 5 \\
\hline 6 & Poor & 38.6 & 29.7 & 19.6 & 15.7 & 13.6 & Solid & Semi-circular & Irregular & Wk 4-5 & Wk 4 \\
\hline 7 & Poor & 14.2 & 7.7 & 4.2 & - & 1.8 & Solid & Circular & Regular & Wk 5 & Wk 2-3 \\
\hline 8 & Poor & 11.4 & 9.7 & 5.5 & 6.0 & 5.7 & Solid & Circular & Regular & None & Wk 4-5 \\
\hline 9 & Poor & 29.8 & 25.9 & 15.4 & 11.4 & 9.8 & Solid & Circular & Regular & None & Wk 3-4 \\
\hline 10 & Poor & 171.1 & 92.3 & 55.8 & 35.8 & 20.5 & Solid & Polypoid & Regular & Wk 2-3 & None \\
\hline 11 & Poor & 18.7 & 22.9 & 14.4 & 4.7 & 5.9 & Solid & Circular & Irregular & Wk 5 & Wk 2 \\
\hline 12 & Poor & 19.6 & 10.2 & 9.0 & 12.3 & 6.8 & Solid & Semi-circular & Regular & Wk 5 & Wk 2-3 \\
\hline 13 & Poor & 29.1 & 23.2 & 20.8 & 10.4 & 9.5 & Solid & Semi-circular & Regular & Wk 4 & Wk 2 \\
\hline
\end{tabular}

For 3 study patients MRI was not performed at one of the 5 time points.

\section{A. absolute volumes per week}

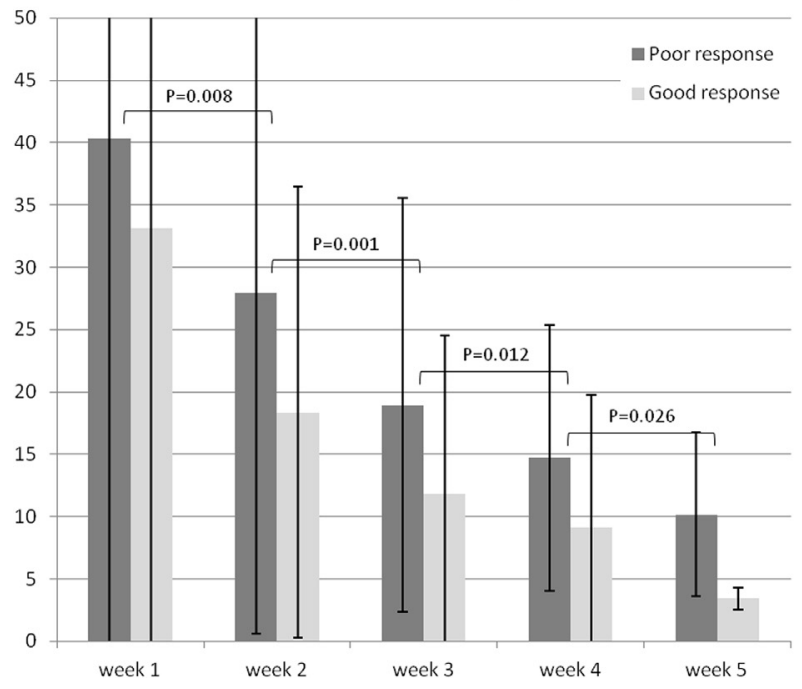

B. volume reduction (\%) per week

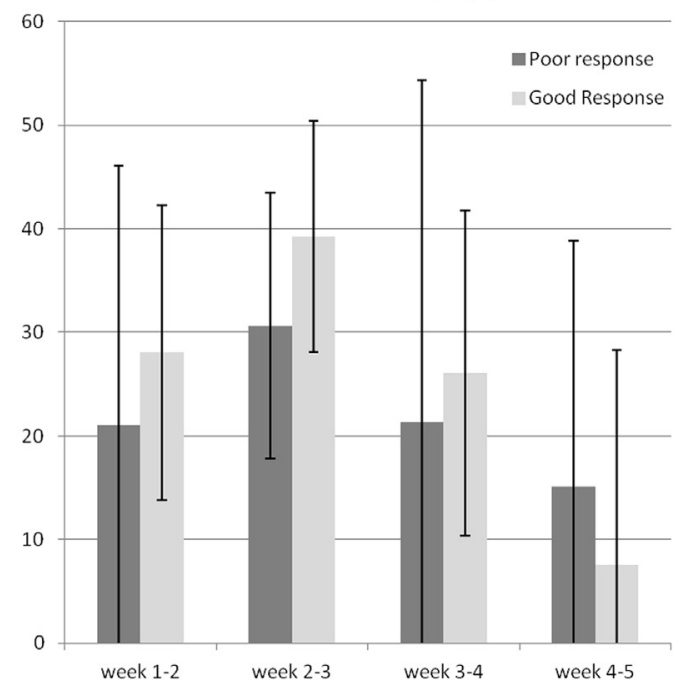

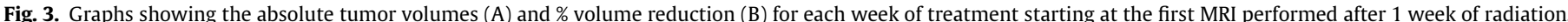

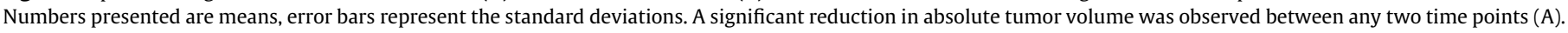

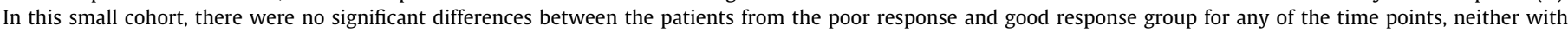
respect to the absolute tumor volume $(A ; P=0.06-0.60)$, nor for the weekly $(B ; P=0.60-1.0)$ volume reduction rates.

For the whole patient group, there was a significant reduction in tumor volume for each time point compared to the previous time point $(P=0.001-0.026)$ with the largest and most significant volume reduction occurring between week 2 and 3 (Fig. $3 a$ and b). The absolute tumor volume was consistently smaller for the good responders, and the relative (\%) decrease in tumor volume for each week was consistently larger for the good responders compared to the poor responders.

\section{Discussion}

Aim of this pilot study was to explore how rectal tumor morphology and volume change during 5 weeks of chemoradiation treatment in order to obtain a better understanding of early signs of rectal tumor regression. Our results show that quantitatively measured rectal tumor volumes decrease significantly during the first five weeks of radiation. Furthermore, our results suggest that early signs of response can also be well appreciated by simple visual assessment where patients that ultimately show a good response tend to show a morphological response - i.e., visually apparent volume reduction and fibrotic transformation - already after the first two to three weeks of treatment, while poor responding tumors tend to show the first visual signs of response later (after the fourth to fifth week) or not all.

Our results with respect to quantitative tumor volumetry are largely in line with previous reports. In a recently published study from 2017, van de Begin et al. performed sequential MRIs before, during and after preoperative chemoradiotherapy in a similar small cohort of 15 locally advanced rectal cancer patients. Although the authors did not make a distinction between good and poor responding tumors they did find - similar to our results - that rectal tumor volumes decreased significantly after each week of radiation. The main rectal tumor regression in their study occurred during the first half of CRT, with speed of shrinkage decreasing over the course [17]. In our study, the first volume measurements were done after the first week of radiation, which unfortunately does not allow an assessment of volume reduction in the first treatment week. Nevertheless our results - like those 
of van de Begin et al - suggest that volume regression is more pronounced early during CRT (with a peak between week 2 and 3 in our cohort). In addition, there are some studies that subjected rectal cancer patients to MRI at only a single or two time points early during radiation. Although the patient cohorts in all these studies were also small (ranging between $n=10-37$ ), they all showed that a significant decrease in tumor volume can already be observed after two (or even one) weeks. Kim et al. evaluated rectal tumor volumes at the end of the second week of treatment. Median decrease in volume was larger for patients with a final histopathological TRG of 1-2 (30.6\%) compared to patients with a TRG score of 3-5 (27.2\%), but the difference - like in our study - did not reach statistical significance, which may very well be related to the small sample size. However, the volume reduction at the end of week two did differ significantly when the final response was defined more arbitrarily as T-downstaging at histopathology compared to the initial T-stage at MRI (cT-stage >ypT-stage), with a larger decrease in volume for the T-downstaged group [13]. Two other groups (Sun et al. and Lambrecht et al.) also used T-downstaging as the main response outcome. Lambrecht et al. measured tumor volume after 10-15 fractions (i.e. 2-3 weeks) and found that the percentage decrease in volume compared to baseline was significantly correlated with T-downstaging [14], while Sun et al. found no significant correlations [10].

In addition to these quantitative study results, our study shows that early signs of response can also be appreciated when visually assessing the images. Morphological MR imaging features of response after completion of CRT are well documented and mainly include a decrease in signal on T2-weighted MRI (indicating fibrotic transformation) and a visually assessable decrease in tumor size [18]. In our experience the same criteria can also be applied to visually assess early tumor response. In the patients from the good response group, an estimated reduction in tumor volume of $\geq 1 / 3$ of the initial tumor volume was typically observed earlier (i.e. after 1-2 weeks) compared to patients from the poor response group who typically showed the first visually apparent volume reduction after 4-5 weeks or not at all during the first five weeks of treatment. Although less obvious, the first signs of fibrosis also tended to occur earlier in the good responders: $75 \%$ of the good responders showed early fibrosis around week $2-3$, while this occurred in only a minority (44\%) of the poor responders. The initial tumor morphology before treatment was largely similar for the two response groups and therefore appears less useful to anticipate the final treatment response.

Altogether, our results as well as results of earlier studies indicate that early signs of response can be captured with routine morphologic imaging both by means of qualitative (visual) interpretation as well as by quantitative tumor volume measurements. These early signs of response may provide an indication of the final response outcome, particularly when assessed mid-CRT, when early tumor regression appears to be most pronounced. This timeline can be of great clinical importance when aiming to identify patients for more personalized neoadjuvant strategies, because it can help in deciding the best time point to evaluate early treatment response. Moreover, if we can identify early during treatment which patients are likely to respond, we can use this information to select those patients who may benefit from further dose escalation to increase the chance of a complete response. In patients unlikely to respond, unnecessary (extra) radiation and thus toxicity may be avoided.

Although outside the scope of this study (which focusses solely on routine T2-weighted MRI), it would be interesting to combine our findings with early changes in 'functional' MR tumor parameters such as those derived from diffusion-weighted or dynamic contrast enhanced MR sequences. Functional sequences are increasingly embedded in research and even clinical rectal MRI protocols and can render quantitative imaging 'biomarkers' reflecting tumor cellularity and perfusion that in various studies have shown promise to assess response after completion of CRT [19-21] as well as to predict response before treatment using measurements derived from the primary staging MRIs [11,19-22]. To date, only a handful of reports have evaluated early changes in functional tumor characteristics early during radiation $[10,12,14]$ and results as to whether these early functional changes provide an added benefit compared to early changes in volume are so far scarce and conflicting. Hence this topic will need to be further investigated in order to confirm findings of previous studies and better understand the complementary value of morphologic and functional imaging parameters for early response assessment.

There are some limitations to our study. First, because our study was intended as an exploratory pilot study, the sample size is small and therefore lacks statistical power. Second, the first study MRI (using the standardized protocol) was performed after the first week of radiation. As a result no quantitative baseline volume measurements were available, which does not allow for volumetric analysis during the first week of radiation. The actual baseline scans performed before the start of treatment were often done in other centers (with different imaging protocols and sequence angulations) and these scans were therefore only used for the visual morphological analyses. Third, the morphological criteria used to assess response - though previously documented in various reports - were more or less subjective, although the readers easily reached consensus in all 13 cases. Second, due to the time consuming methodology, the volumetric measurements were all done by one expert reader. This does not account for interobserver variations, though previous research has shown that tumor volume measurements in rectal cancer are highly reproducible among readers [23-25]. Finally, 3 out of the 13 study patients underwent MRIs at only four instead of all five time points.

In conclusion, the results of this pilot study suggest that significant changes in rectal tumor morphology (i.e. early fibrosis) and volume can already be observed early during preoperative radiation, both when measured quantitatively and when assessed visually. These changes appear to be indicative of the final treatment outcome and - although no firm conclusions can be drawn yet - most pronounced during the first half (second to third week) of treatment. Next steps would be to assess the value of adding functional imaging information and to validate our findings in larger cohorts in order to establish the best imaging sequences, criteria and particularly time point for early treatment response evaluation so that these may be used to further personalize neoadjuvant treatment and select patients for dose escalation to maximize response rates.

\section{Conflict of interest}

None of the authors have any conflicts of interest to disclose.

\section{References}

[1] Martens MH, Maas M, Heijnen LA, et al. Long-term outcome of an organ preservation program after neoadjuvant treatment for rectal cancer. J Natl Cancer Inst 2016;108(12).

[2] Appelt AL, Ploen J, Harling H, et al. High-dose chemoradiotherapy and watchful waiting for distal rectal cancer: a prospective observational study. Lancet Oncol 2015;16:919-27.

[3] Habr-Gama A, Gama-Rodrigues J, Sao Juliao GP, et al. Local recurrence after complete clinical response and watch and wait in rectal cancer after neoadjuvant chemoradiation: impact of salvage therapy on local disease control. Int J Radiat Oncol Biol Phys 2014;88:822-8.

[4] Smith JD, Ruby JA, Goodman KA, et al. Nonoperative management of rectal cancer with complete clinical response after neoadjuvant therapy. Ann Surg 2012;256:965-72. 
[5] Maas M, Nelemans PJ, Valentini V, et al. Long-term outcome in patients with a pathological complete response after chemoradiation for rectal cancer: a pooled analysis of individual patient data. Lancet Oncol 2010;11: $835-44$.

[6] Burbach JP, den Harder AM, Intven M, van Vulpen M, Verkooijen HM, Reerink O. Impact of radiotherapy boost on pathological complete response in patients with locally advanced rectal cancer: a systematic review and meta-analysis. Radiother Oncol 2014;113:1-9.

[7] Li C, Lan X, Yuan H, Feng H, Xia X, Zhang Y. 18F-FDG PET predicts pathological response to preoperative chemoradiotherapy in patients with primary rectal cancer: a meta-analysis. Ann Nucl Med 2014;28:436-46.

[8] Zhang C, Tong J, Sun X, Liu J, Wang Y, Huang G. 18F-FDG-PET evaluation of treatment response to neo-adjuvant therapy in patients with locally advanced rectal cancer: a meta-analysis. Int J Cancer 2012;131:2604-11.

[9] Lambrecht M, Deroose C, Roels S, et al. The use of FDG-PET/CT and diffusionweighted magnetic resonance imaging for response prediction before, during and after preoperative chemoradiotherapy for rectal cancer. Acta Oncol 2010;49:956-63.

[10] Sun YS, Zhang XP, Tang L, et al. Locally advanced rectal carcinoma treated with preoperative chemotherapy and radiation therapy: preliminary analysis of diffusion-weighted MR imaging for early detection of tumor histopathologic downstaging. Radiology 2010;254:170-8.

[11] Barbaro B, Vitale R, Valentini V, et al. Diffusion-weighted magnetic resonance imaging in monitoring rectal cancer response to neoadjuvant chemoradiotherapy. Int J Radiat Oncol Biol Phys 2012;83:594-9.

[12] Dijkhoff RAP, Beets-Tan RGH, Lambregts DMJ, Beets GL, Maas M. Value of DCEMRI for staging and response evaluation in rectal cancer: A systematic review. Eur J Radiol 2017;95:155-68.

[13] Kim YC, Lim JS, Keum KC, et al. Comparison of diffusion-weighted mri and mr volumetry in the evaluation of early treatment outcomes after preoperative chemoradiotherapy for locally advanced rectal cancer. J Magn Reson Imaging 2011.

[14] Lambrecht M, Vandecaveye $\mathrm{V}$, De Keyzer $\mathrm{F}$, et al. Value of diffusion-weighted magnetic resonance imaging for prediction and early assessment of response to neoadjuvant radiochemotherapy in rectal cancer: preliminary results. Int J Radiat Oncol Biol Phys 2012;82:863-70.
[15] van den Begin R, Kleijnen J-P, Engels B, et al. Tumor volume regression during preoperative chemoradiotherapy for rectal cancer: a prospective observational study with weekly MRI. Acta Oncol 2017:1-5.

[16] Fedorov A, Beichel R, Kalpathy-Cramer J, et al. 3D Slicer as an image computing platform for the Quantitative Imaging Network. Magn Reson Imaging 2012;30:1323-41.

[17] Mandard AM, Dalibard F, Mandard JC, et al. Pathologic assessment of tumor regression after preoperative chemoradiotherapy of esophageal carcinoma Clinicopathologic correlations. Cancer 1994;73:2680-6.

[18] Barbaro B, Vitale R, Leccisotti L, et al. Restaging locally advanced rectal cance with MR imaging after chemoradiation therapy. Radiographics 2010;30: 699-716.

[19] Intven M, Monninkhof EM, Reerink O, Philippens ME. Combined T2w volumetry, DW-MRI and DCE-MRI for response assessment after neoadjuvant chemoradiation in locally advanced rectal cancer. Acta Onco 2015:54:1729-36.

[20] Martens MH, Subhani S, Heijnen LA, et al. Can perfusion MRI predict response to preoperative treatment in rectal cancer? Radiother Oncol 2015;114: 218-23.

[21] Jung SH, Heo SH, Kim JW, et al. Predicting response to neoadjuvant chemoradiation therapy in locally advanced rectal cancer: diffusionweighted 3 Tesla MR imaging. J Magn Reson Imaging 2012;35:110-6.

[22] George ML, Dzik-Jurasz AS, Padhani AR, et al. Non-invasive methods of assessing angiogenesis and their value in predicting response to treatment in colorectal cancer. Br J Surg 2001;88:1628-36.

23] Curvo-Semedo L, Lambregts DM, Maas M, et al. Rectal cancer: assessment of complete response to preoperative combined radiation therapy with chemotherapy-conventional MR volumetry versus diffusion-weighted MR imaging. Radiology 2011;260:734-43.

[24] Martens MH, van Heeswijk MM, van den Broek JJ, et al. Prospective, multicenter validation study of magnetic resonance volumetry for response assessment after preoperative chemoradiation in rectal cancer: can the results in the literature be reproduced? Int J Radiat Oncol Biol Phys 2015;93:1005-14.

[25] Petrillo M, Fusco R, Catalano O, et al. MRI for assessing response to neoadjuvant therapy in locally advanced rectal cancer using DCE-MR and DW-MR data sets: a preliminary report. Biomed Res Int 2015;2015:514740. 